\title{
Selectivity of beta-blockers, cardiovascular and all-cause mortality in people with hypoglycaemia: an observational study
}

Zaccardi F ${ }^{a}$

Nystrup Husemoen L L ${ }^{b}$

Thorsted B L ${ }^{b}$

Webb D R a

Paul S K ${ }^{\mathrm{c}}$

Davies M J

Khunti $\mathrm{K}^{\mathrm{a}}$

a Diabetes Research Centre, University of Leicester, Gwendolen Rd, Leicester LE5 4PW, UK

${ }^{b}$ Novo Nordisk A/S, Vandtårnsvej a08, b860 Søborg, Denmark

' Melbourne EpiCentre, University of Melbourne and Melbourne Health, Level 7, East, c00 Grattan Street, Parkville Victoria, Melbourne, Australia

Corresponding Author

Dr Francesco Zaccardi

Leicester Diabetes Centre, Leicester General Hospital

Leicester, Leicestershire, LE5 4PW, England, UK

Phone: +44 01162584389 - Fax: +44 01162584499

Email: frazac@fastwebnet.it

Keywords: Beta-blockers; cardiovascular disease; electronic health records; hypoglycemia; mortality

\section{Abbreviations/Acronyms}

Cl Confidence interval

CPRD Clinical Practice Research Datalink

HES Hospital Episode Statistics

ICD-10 International Statistical Classification of Diseases and Related Health Problems - 10th Revision

ONS Office for National Statistics

RCT Randomised controlled trial

SD Standard deviation 


\begin{abstract}
Background and Aims: The association of beta-blockers and their selectivity with mortality and cardiovascular events in patients with and without hypoglycaemia is unknown.
\end{abstract}

Methods and Results: Insulin-treated patients with diabetes were identified within the UK CPRD database. All-cause deaths, cardiovascular events, and hypoglycaemic episodes were captured to assess the interaction between beta-blocker therapy and selectivity with hypoglycaemia. 13682 patients, of which 2036 (14.9\%) with at least one hypoglycaemic episode, were included; 3148 deaths and 1235 cardiovascular events were recorded during a median of 2.3 and 4.7 years in patients with and without incident hypoglycaemia, respectively. Treatment with any beta-blocker was not associated with risk of death in both patients with and without hypoglycaemia, without significant interaction. Compared to no therapy, non-selective beta-blockers were associated with higher risk of death in patients without hypoglycaemia (hazard ratio (HR) 2.93 [1.26-6.83] in the fully adjusted model) but not in those with hypoglycaemia; interactions was not significant. For beta1-selective betablockers, there was no association with mortality in both patients with and without hypoglycaemia, without significant interaction. After missing data imputation, results were consistent for nonselective beta-blockers (HR in patients without hypoglycaemia 1.59 [1.22-2.08]) while indicated a reduced risk of death for beta1-selective beta-blockers in patients with hypoglycaemia (HR 0.76 [0.610.94]). Due to few cardiovascular events, complete-case analysis compared only any vs no betablocker therapy and indicated no associations with therapy or interaction by hypoglycaemia.

Conclusion: In patients with hypoglycaemic episodes, treatment with beta1-selective beta-blockers may potentially reduce the risk of death. These explorative findings and the potential role of confounding by indication need to be evaluated in other studies. 


\section{INTRODUCTION}

Hypoglycaemia is the most common complication of glucose-lowering pharmacological treatments in diabetes mellitus [1]. In the last decade, post-hoc analyses of randomised controlled trials (RCTs) and meta-analyses of observational studies have suggested an increased risk of all-cause and cardiovascular mortality in intensively-treated type 2 diabetes patients who experience higher rates of severe hypoglycaemia, [2]. The epidemiological evidence tallies with increasing experimental observation of multiple deleterious effects of hypoglycaemia on the cardiovascular system. A state of controlled hypoglycaemia results in arrhythmogenic changes potentially leading to bradycardia, tachycardia, ventricular fibrillation, and sudden death (fatal arrhythmias) [3]. Such abnormalities are mediated by sympathoadrenal (adrenergic) activation [4], as they are markedly prevented by pharmacological beta-adrenergic blockade [5].

These pathophysiological mechanisms would suggest a possible modification of the relationship between hypoglycaemia and cardiovascular events by beta-blocker therapy. Moreover, as the cardiac effects of beta-blockers are mainly mediated by beta1 receptors, the predominant receptor type in the heart (Supplemental Material Table S1), beta1-selective beta-blockers could have a greater inhibitory effect on hypoglycaemia-induced arrhythmias compared to non-selective beta-blockers. Non-selective beta-blockers are also more likely to inhibit liver glycogenolysis and gluconeogenesis through the beta2-receptor and therefore theoretically increase the risk of glycopenia-related tissue damage.

In this observational study, we examined whether the associations between hypoglycaemia and risk of cardiovascular and all-cause mortality was modified by treatment with any beta-blocker and according to their selectivity. 


\section{METHODS}

\section{Data}

We used data from the Clinical Practice Research Datalink (CPRD) database which includes anonymized longitudinal records of about 7\% of the UK population [6]. Patient-level demographic, clinical, laboratory, and prescription records are collected following an agreed protocol and are regularly linked to Hospital Episode Statistics (HES) data, which contains details of all hospital admissions, outpatient appointments, and emergency attendances at National Health Service hospitals in England; and to the Office of National Statistics (ONS), which includes information on the official date and cause of death. This study was conducted following a pre-specified protocol approved by an Independent Scientific Advisory Committee (16_104). Codes used to identify the population, main exposures and covariates, and outcomes are reported in Table S2.

\section{Cohort definition}

The study design is graphically shown in Figure $\mathbf{S 1}$ in the Supplemental Material. We constructed a retrospective cohort consisting of all insulin-treated patients, 30 years of age or older, with a diagnosis of type 1 or type 2 diabetes between 1/1/2001 and 31/12/2007; patients were classified into diabetes types based on a combination of Read codes, therapy, and age at diagnosis $[7,8]$. We selected patients on insulin treatment as they have an increased risk of hypoglycaemia [9]. The index date was the start of insulin treatment (identified by the second insulin prescription) after 1/1/2001.

\section{Main exposures and covariates}

Data on hypoglycaemic episodes were obtained via ICD-10 codes for HES (E16.0, E16.1, and E16.2) and Read/OXMIS codes for CPRD (Table S2) [7]. All hypoglycaemic episodes recorded in HES were severe (i.e., requiring hospital admission), whereas those in the CPRD could be severe or non-severe; patients with hypoglycaemic episode(s) before the initiation of insulin therapy were excluded. Patients were considered unexposed until the first post-baseline episode of hypoglycaemia, if it occurred, and 
exposed thereafter (Figure S1). Data on HbA1c, blood lipids, smoking status, body mass index, systolic blood pressure, Charlson comorbidity score, socio-economic status, prevalent cardiovascular disease, sex, and age were assessed six months around the index date. Use of beta-blockers was ascertained three months around the index date; in patients experiencing hypoglycaemia, treatment needed to be present within three months after the episode as therapy could be suspended following hypoglycaemia. Beta-blockers were determined using prescribing data and included all drugs classified in the British National Formulary [10]; we considered two exposures: any beta-blocker and betablocker according to their cardio-selectivity (beta1-selective and non-selective beta-blockers) [10].

\section{Outcomes}

For both exposed and unexposed time, patients were followed until the date of the first event (death or non-fatal myocardial infarction or stroke) or were otherwise censored (transferring out of practice, last collection of data from practice, end of study on 31/12/2010). We defined three outcomes: allcause death; a composite endpoint of major cardiovascular events (fatal or non-fatal myocardial infarction or stroke); and single cardiovascular endpoints. Patients were considered as having prevalent cardiovascular disease if there was a registered cardiovascular event code in HES or CPRD before index date.

\section{Statistical analysis}

Details on power calculation are reported in the supplementary material. We summarised the characteristics of included patients as mean and standard deviation (SD) or median and interquartile range for continuous data and as number and percentage for categorical data.

The analytical plan is shown in Table S3. We planned to investigate the association between use of beta-blocker and outcomes using Cox proportional hazard models progressively adjusted for potential confounders and including an interaction term (beta-blocker*hypoglycaemia) to explore whether the effect of beta-blocker was modified by hypoglycaemic events. In the main analysis, we used all non- 
missing data (i.e., complete-case), any beta-blockers and beta-blockers according to their selectivity as exposure, and all-cause death as outcome. The main analysis was repeated after multiple multivariate imputation of missing values with 10 imputations by applying Rubin's rule. In secondary analyses, we planned to investigate the associations of any beta-blockers and beta-blockers according to their selectivity with cardiovascular outcomes (composite and each cardiovascular endpoints) using complete-case regressions. At reviewing stage, we also estimated the association between betablockers selectivity and risk of hypoglycaemia.

Analyses were performed with Stata MP (v. 14.1) and results are reported with robust $95 \%$ confidence interval $(\mathrm{Cl})$ to account for the presence of clusters (i.e., same patients before and after hypoglycaemia); a p-value $<0.05$ was deemed statistically significant. 


\section{RESULTS}

\section{Participants and exposures}

The study included 13682 patients (6035 [44.1\%] women; mean \pm SD age: $62 \pm 14$ ) of which 2036 (14.9\%) had at least one episode of hypoglycaemia, six at the index date and 2030 episodes during the follow-up. The pattern of missing data for individual patients is shown in Figure S2: data were complete for socioeconomic status, prevalent cardiovascular disease, sex, and age, and virtually complete for Charlson score (33; $0.2 \%$ missing).

Characteristics of patients, by incident hypoglycaemia and beta-blocker use, are reported in Table 1: compared to subjects without beta-blockers, those on any beta-blocker where older, had a higher body mass index and Charlson score, a similar systolic blood pressure, HbA1c and HDL-cholesterol, and a lower LDL-cholesterol; beta-blockers were more commonly prescribed in men, patients with prevalent cardiovascular disease, and previous or never smokers; less marked differences were observed comparing patients on beta1-selective vs non-selective beta-blockers (Table S4). In both patients with and without incident hypoglycaemia, the most common beta-blockers were atenolol (48.7\% and 63.6\%, respectively) and bisoprolol (36.1\% and 23.0\%, respectively) (Figure S3).

\section{Events and associations}

Main analysis

During a median follow-up of 2.34 years (5996.7 person-years) in patients with incident hypoglycaemia and 4.65 years (64619.2 person-years) in those without, 3148 all-cause death, 1235 composite cardiovascular outcome, 664 non-fatal myocardial infarction, and 488 non-fatal stroke events were recorded (Table 2).

In patients without hypoglycaemia, adjusting for age and sex differences, the risk of all-cause death was not different comparing any beta-blocker vs no beta-blocker (hazard ratio (HR): $1.06 ; 95 \% \mathrm{Cl}$ : 0.97-1.16; Figure 1); a qualitative different result was found in patients with hypoglycaemia, being 
the corresponding $\mathrm{HR} 0.85$ (95\% $\mathrm{Cl}: 0.70-1.05)$ and $\mathrm{p}=0.057$ for interaction comparing the effect of any beta-blocker in patients with and without hypoglycaemia. In models progressively adjusted for other confounders, results indicated no associations of any beta-blocker with the risk of mortality in both patients with and without hypoglycaemia, without significant interactions (Figure 1).

For non-selective beta-blockers, the risk of death was consistently higher compared to no therapy in patients without hypoglycaemia for all models, ranging from HR 1.94 (95\% $\mathrm{Cl}$ : 0.70-1.05) accounting only for age and sex to $2.93(1.26-6.83)$ in the fully adjusted model (Figure 1); conversely, no differences were observed in patients with hypoglycaemia. P-values for interactions were not significant in all models.

In patients on selective beta1-selective beta-blockers, the risk of death was not different comparing use vs no use in both patients with and without hypoglycaemia; P-values for interaction were not significant in all models (Figure 1).

\section{Multiple imputation analysis}

After imputation of missing data, in patients without hypoglycaemia the risk of death was not different comparing any beta-blocker (HR 0.96; $95 \% \mathrm{Cl}$ : $0.87-1.05)$ or beta1-selective beta-blocker $(0.92 ; 0.84-$ 1.01) vs no therapy in fully adjusted models; conversely, the risk was higher for non-selective betablockers (1.59; 1.22-2.08; Figure 2). In a similar model, in patients with hypoglycaemia the risk of death was lower comparing any beta-blocker (HR 0.76 ; $95 \% \mathrm{Cl}$ : $0.62-0.94)$ or beta1-selective betablocker $(0.76 ; 0.61-0.94)$ vs no therapy. P-value for interaction was significant only for any betablocker $(P=0.049)$.

\section{Secondary analyses}

Given the low number of patients and events, secondary analyses were only performed for the combined cardiovascular endpoint fatal or non-fatal myocardial infarction or stroke using any betablocker as exposure. In a complete-case investigation, the risk of the composite outcome was higher 
in patients without hypoglycaemia accounting for age and sex (HR 1.49; $95 \% \mathrm{Cl}$ : 1.31-1.70); the association persisted upon further adjustment for smoking status and systolic blood pressure (1.54; 1.23-1.93) but was not present including other potential confounders (Figure S4). There was no association between use of any beta-blocker and the composite cardiovascular outcome for patients with hypoglycaemia in all models (Figure S4); P-values for interactions were not significant in all models.

Beta-blocker selectivity and risk of hypoglycaemia

We did not find evidence of an increased risk of hypoglycaemia in non-selective vs beta1-selective beta-blockers. Age and sex adjusted hazard ratio was 1.07 (95\% Cl: 0.66-1.71) and, upon inclusion of systolic blood pressure and smoking, $1.12(0.46-2.75)$. Few participants and events did not allow further adjustments. 


\section{DISCUSSION}

In this observational study, beta1-selective beta-blockers were associated with a possible reduction of mortality in patients with episodes of hypoglycaemia; conversely, non-selective beta-blockers were associated with an increased risk in patients without hypoglycaemia. Given the limited numbers by beta-blocker selectivity, findings for the composite endpoint of fatal or non-fatal myocardial infarction or stroke are more difficult to interpret, yet we observed no associations for any beta-blocker in both patients with and without hypoglycaemia.

Clear evidence of cardiovascular and mortality benefit of beta-blockers in patients with diabetes is lacking. A recent position statement suggests beta-blockers use in patients with coronary disease or heart failure, yet they have not been shown to reduce mortality as blood pressure-lowering agents in the absence of these conditions [11-13]. Moreover, a recent prospective cohort study has reported a $50 \%$ increased mortality in patients with diabetes taking any beta-blocker or beta1-selective betablocker compared to no beta-blocker, irrespective of concomitant coronary heart disease [14]. These findings confirmed a previous post-hoc analysis of around 5000 participants of the ACCORD RCT, which showed a $30 \%$ to $50 \%$ increased risk of cardiovascular events in participants with type 2 diabetes on any beta-blocker compared to no beta-blocker, independent of the presence of coronary heart disease or heart failure [15]. Notably, there was no interaction between intensive glucose control and betablocker therapy on the risk of cardiovascular events, cardiovascular mortality, and all-cause mortality, suggesting a similar effect of beta-blockers irrespective of treatment randomisation (intensive vs routine) [16]. As participants in the ACCORD intensive arm experienced higher rates of severe hypoglycaemia [17], these results question the indication to re-consider or suspend beta-blockers in patients at high risk of hypoglycaemia. In another epidemiological investigation of a different RCT (BARI 2D), it was conversely observed a potential reduction of all-cause mortality associated with any beta-blocker therapy in type 2 diabetes patients who underwent early revascularization, without a clear difference between patients with or without myocardial infarction and/or heart failure with 
reduced left ventricular ejection fraction; less clear were the overall effects of any beta-blocker on other outcomes, including cardiovascular death and fatal or non-fatal myocardial infarction or stroke. More recently, in a study of around 1800 patients with heart failure, beta-blocker therapy (mainly bisoprolol) was associated with a greater mortality reduction in patients with type 2 diabetes compared to those without [18].

Available data are similarly conflicting about the relationship between beta-blocker therapy and risk of hypoglycaemia. From a clinical perspective, beta-blockers have been considered a potential problem as they could mask the symptoms of hypoglycaemia. In an observational study of nearly one million adults with diabetes, the unadjusted rates of severe hypoglycaemia resulting in emergency department visit or hospital admission were around two-times higher in patients taking any betablocker vs no beta-blocker [19]. However, in a post-hoc analysis of the ACCORD, the relative risk of severe hypoglycaemia was much smaller, being around 1.3-times higher in patients taking any betablocker vs no beta-blocker [15]; notably, there was no interaction between type of intervention (intensive vs routine) and use of beta-blocker on the risk of severe hypoglycaemia [16].

In contrast with the above findings, the available evidence relating to hypoglycaemia and risk of longterm outcomes, such as cardiovascular events and mortality, is more consistent. Initial observations were mainly from epidemiological analyses of large RCTs, which showed a higher mortality in patients experiencing severe hypoglycaemia [2, 20-22]. Further evidence has progressively emerged from other post-hoc analyses of RCTs [23], meta-analyses [24], and observational studies. In large cohorts severe hypoglycaemia has been linked to all-cause and cause-specific mortality [7, 25-27]: it remains uncertain, however, whether hypoglycaemia is causally linked to the outcomes or simply a marker of vulnerability (i.e., a confounder).

While previous studies did not examine potential differences by beta-blocker selectivity, we specifically explored interaction according to beta-blocker pharmacodynamics. Investigating potential divergent effects by beta-blocker selectivity is relevant in view of the pathophysiological and pharmacological implications of hypoglycaemia-driven sympathoadrenal response. As the cardiac 
effects of catecholamines are mainly mediated by beta1 receptors, beta1-selective beta-blockers could potentially reduce the risk of cardiac electrophysiological abnormalities. Our results did not show "formal" statistically significant interactions at the a-priori defined significance level of 0.05 , possibly because of the low number of patients with incident hypoglycaemia, particularly for nonselective beta-blockers. However, given the explorative nature of the study, we believe that less emphasis should be placed on the statistical significance level and more focus, instead, on the clinical importance of the results. At the same time, we also deem that the possible beneficial effect of beta1selective beta-blockers should be further explored in other studies, due to the potentially relevant clinical implications.

To our knowledge, this is the first large observational study to investigate beta-blockers and their selectivity in the association of hypoglycaemia with cardiovascular and all-cause mortality. In the analyses, we accounted for multiple potential confounders and considered as exposures all hypoglycaemic episodes, while most of previous epidemiological analyses focused only on severe episodes. There are also some limitations. In pharmacoepidemiological studies, confounding by indication is a potential bias which may result in inaccurate treatment-outcome causal estimates. Among the multiple analytical methods to control for this confounding, we opted for regression adjustment which included factors related to both the outcome (all-cause death and cardiovascular outcomes) and the exposure/treatment (beta-blockers) and are not intermediate between the treatment and the outcome [28]. In particular, models included prevalent cardiovascular disease as it could act as a confounder between the prescription of some beta-blockers (for example, selective beta-blockers are more likely prescribed for myocardial infarction while non-selective for noncardiovascular conditions) and risk of death. There is the possibility, however, that other potential confounders have not been accounted for in these analyses. Although the availability and the quality of data have improved after 2004 [6], for some variables data were not collected (i.e., kidney function) or were unavailable in a substantial proportion of patients, which could have impacted on risk estimates. To reduce the risk of this bias, we performed multiple imputation analyses. We could not 
explore if a divergent relationship exist, comparing beta1-selective vs non-selective beta-blockers, when the outcome is cardiovascular instead of all-cause mortality: this was due to the small number of patients, in particular for non-selective beta-blockers, and events, which restricted the analysis of combined cardiovascular endpoint only to any beta-blocker. For the same reason, it was not possible to investigate associations with individual cardiovascular endpoints and stratify the analysis by prevalent cardiovascular disease or diabetes type. Most non-severe hypoglycaemic events are selftreated by patients and likely to be unreported to physicians; moreover, general practices participating in the CPRD report disease events better than those not participating [29]. Lastly, data on hypoglycaemia unawareness were not available; the sample of patients with diabetes was defined only including those with Read codes in $\mathrm{C} 10+$; information on covariates were extracted only at index date; and, due to the observational nature of the investigation, no causality can be directly inferred from these findings.

The increasing prevalence of aging, multimorbid patients with diabetes has resulted in global increasing trends of hypoglycaemia and hypoglycaemia-related mortality [30, 31]; approaches to reduce the burden of hypoglycaemia-related death are therefore warranted. Our data indicate that non-selective beta-blockers may increase the risk of mortality in patients without hypoglycaemia; conversely, in patients with hypoglycaemia, there was no evidence of an increased risk for nonselective beta-blockers and a potentially reduced risk for beta1-selective beta-blockers. These results, however, need to be confirmed in future larger studies to determine whether beta1-selective betablockers should be prescribed in patients with frequent hypoglycaemic episodes to reduce the risk of death and cardiovascular events. 


\section{Acknowledgements}

FZ, MJD, and KK acknowledge the NIHR CLAHRC - EM and the NIHR Leicester Biomedical Research Centre. This report is an independent research funded by the National Institute for Health Research. The views expressed in this publication are those of the author(s) and not necessarily those of the NHS, the National Institute for Health Research or the Department of Health. Data access was granted as part of the CPRD licence agreement hold by Novo Nordisk A/S. SKP acknowledges the support from the Australian Government's National Collaborative Research Infrastructure Strategy (NCRIS) initiative through Therapeutic Innovation Australia.

\section{Funding}

This study has been supported by MRC Proximity to Discovery: Industry Engagement Fund, 2017. FZ is funded with an unrestricted educational grant from the National Institute for Health Research Collaboration for Leadership in Applied Health Research and Care (CLAHRC) East Midlands to the University of Leicester.

\section{Declaration of interests}

KK has acted as a consultant and speaker for Novartis, Novo Nordisk, Sanofi-Aventis, Lilly and Merck Sharp \& Dohme. He has received grants in support of investigator and investigator initiated trials from Novartis, Novo Nordisk, Sanofi-Aventis, Lilly, Pfizer, Boehringer Ingelheim and Merck Sharp \& Dohme. KK has received funds for research, honoraria for speaking at meetings and has served on advisory boards for Lilly, Sanofi-Aventis, Merck Sharp \& Dohme and Novo Nordisk. MJD has acted as consultant, advisory board member and speaker for Novo Nordisk, Sanofi-Aventis, Lilly, Merck Sharp \& Dohme, Boehringer Ingelheim, AstraZeneca and Janssen, an advisory board member for Servier and as a speaker for Mitsubishi Tanabe Pharma Corporation and Takeda Pharmaceuticals International Inc. She has received grants in support of investigator and investigator initiated trials from Novo Nordisk, Sanofi-Aventis, Lilly, Boehringer Ingelheim and Janssen. DRW has received grant in support of investigator initiated studies and honoraria from Sanofi-Aventis and Novo Nordisk. LLNH is an employee a Novo Nordisk A/S. BLT is Novo Nordisk A/S employee and shareholder. SKP has acted as a consultant and/or speaker for Novartis, GI Dynamics, Roche, AstraZeneca, Guangzhou Zhongyi Pharmaceutical and Amylin Pharmaceuticals LLC. He has received grants in support of investigator and investigator initiated clinical studies from Merck, Novo Nordisk, AstraZeneca, Hospira, Amylin Pharmaceuticals, Sanofi-Avensis and Pfizer. FZ: None.

\section{Data availability}

CPRD data governance does not allow us to distribute patient data to other parties. Researchers may apply for data access at http://www.CPRD.com/. Statistical codes are available on request from the corresponding author (FZ). 


\section{REFERENCES}

[1] International Hypoglycaemia Study Group. Minimizing Hypoglycemia in Diabetes. Diabetes Care. 2015;38:1583-91. doi: 10.2337/dc15-0279

[2] Pistrosch F, Hanefeld M. Hypoglycemia and Cardiovascular Disease: Lessons from Outcome Studies. Curr Diab Rep. 2015;15:117. doi: 10.1007/s11892-015-0678-2

[3] Chow E, Bernjak A, Williams S, Fawdry RA, Hibbert S, Freeman J, et al. Risk of cardiac arrhythmias during hypoglycemia in patients with type 2 diabetes and cardiovascular risk. Diabetes. 2014;63:1738-47. doi: 10.2337/db13-0468

[4] Tsujimoto T, Yamamoto-Honda R, Kajio H, Kishimoto M, Noto H, Hachiya R, et al. Effectiveness of Prior Use of Beta-Blockers for Preventing Adverse Influences of Severe Hypoglycemia in Patients With Diabetes: An Observational Study. Medicine (Baltimore). 2015;94:e1629. doi: 10.1097/MD.0000000000001629

[5] Reno CM, Daphna-Iken D, Chen YS, VanderWeele J, Jethi K, Fisher SJ. Severe hypoglycemiainduced lethal cardiac arrhythmias are mediated by sympathoadrenal activation. Diabetes. 2013;62:3570-81. doi: 10.2337/db13-0216

[6] Herrett E, Gallagher AM, Bhaskaran K, Forbes H, Mathur R, van Staa T, et al. Data Resource Profile: Clinical Practice Research Datalink (CPRD). Int J Epidemiol. 2015;44:827-36. doi: 10.1093/ije/dyv098

[7] Khunti K, Davies M, Majeed A, Thorsted BL, Wolden ML, Paul SK. Hypoglycemia and risk of cardiovascular disease and all-cause mortality in insulin-treated people with type 1 and type 2 diabetes: a cohort study. Diabetes Care. 2015;38:316-22. doi: 10.2337/dc14-0920

[8] Owusu Adjah ES, Montvida O, Agbeve J, Paul SK. Data Mining Approach to Identify Disease Cohorts from Primary Care Electronic Medical Records: A Case of Diabetes Mellitus. The Open Bioinformatics Journal 2017;10:16-27. doi:

[9] Edridge CL, Dunkley AJ, Bodicoat DH, Rose TC, Gray L, Davies MJ, et al. Prevalence and Incidence of Hypoglycaemia in 532,542 People with Type 2 Diabetes on Oral Therapies and Insulin: A Systematic Review and Meta-Analysis of Population Based Studies. PLoS One. 2015;10:e0126427. doi: 10.1371/journal.pone.0126427

[10] British National Formulary; Edition 70, September 2015-March 2016; Chapter 2.4, pp. 139-147.

[11] Ettehad D, Emdin CA, Kiran A, Anderson SG, Callender T, Emberson J, et al. Blood pressure lowering for prevention of cardiovascular disease and death: a systematic review and meta-analysis. Lancet. 2016;387:957-67. doi: 10.1016/S0140-6736(15)01225-8

[12] de Boer IH, Bangalore S, Benetos A, Davis AM, Michos ED, Muntner P, et al. Diabetes and Hypertension: A Position Statement by the American Diabetes Association. Diabetes Care. 2017;40:1273-84. doi: 10.2337/dci17-0026

[13] American Diabetes Association. Introduction: Standards of Medical Care in Diabetes-2018. Diabetes Care. 2018;41:S1-S2. doi: 10.2337/dc18-Sint01

[14] Tsujimoto T, Kajio H, Shapiro MF, Sugiyama T. Risk of All-Cause Mortality in Diabetic Patients Taking beta-Blockers. Mayo Clin Proc. 2018;93:409-18. doi: 10.1016/j.mayocp.2017.11.019

[15] Tsujimoto T, Sugiyama T, Shapiro MF, Noda M, Kajio H. Risk of Cardiovascular Events in Patients With Diabetes Mellitus on beta-Blockers. Hypertension. 2017;70:103-10. doi:

10.1161/HYPERTENSIONAHA.117.09259 
[16] Tsujimoto T, Sugiyama T, Noda M, Kajio H. Intensive Glycemic Therapy in Patients With Type 2 Diabetes on beta-Blockers. Diabetes Care. 2016;39:1818-26. doi: 10.2337/dc16-0721

[17] Gerstein HC, Miller ME, Byington RP, Goff DC, Jr., Bigger JT, Buse JB, et al. Effects of intensive glucose lowering in type 2 diabetes. N Engl J Med. 2008;358:2545-59. doi: 10.1056/NEJMoa0802743

[18] Witte KK, Drozd M, Walker AMN, Patel PA, Kearney JC, Chapman S, et al. Mortality Reduction Associated With beta-Adrenoceptor Inhibition in Chronic Heart Failure Is Greater in Patients With Diabetes. Diabetes Care. 2018;41:136-42. doi: 10.2337/dc17-1406

[19] Pathak RD, Schroeder EB, Seaquist ER, Zeng C, Lafata JE, Thomas A, et al. Severe Hypoglycemia Requiring Medical Intervention in a Large Cohort of Adults With Diabetes Receiving Care in U.S. Integrated Health Care Delivery Systems: 2005-2011. Diabetes Care. 2016;39:363-70. doi: $10.2337 / \mathrm{dc} 15-0858$

[20] Bonds DE, Miller ME, Bergenstal RM, Buse JB, Byington RP, Cutler JA, et al. The association between symptomatic, severe hypoglycaemia and mortality in type 2 diabetes: retrospective epidemiological analysis of the ACCORD study. BMJ. 2010;340:b4909. doi: 10.1136/bmj.b4909

[21] Zoungas S, Patel A, Chalmers J, de Galan BE, Li Q, Billot L, et al. Severe hypoglycemia and risks of vascular events and death. N Engl J Med. 2010;363:1410-8. doi: 10.1056/NEJMoa1003795

[22] Mellbin LG, Ryden L, Riddle MC, Probstfield J, Rosenstock J, Diaz R, et al. Does hypoglycaemia increase the risk of cardiovascular events? A report from the ORIGIN trial. Eur Heart J. 2013;34:313744. doi: 10.1093/eurheartj/eht332

[23] Pieber TR, Marso SP, McGuire DK, Zinman B, Poulter NR, Emerson SS, et al. DEVOTE 3: temporal relationships between severe hypoglycaemia, cardiovascular outcomes and mortality. Diabetologia. 2018;61:58-65. doi: 10.1007/s00125-017-4422-0

[24] Goto A, Arah OA, Goto M, Terauchi Y, Noda M. Severe hypoglycaemia and cardiovascular disease: systematic review and meta-analysis with bias analysis. BMJ. 2013;347:f4533. doi: 10.1136/bmj.f4533

[25] McCoy RG, Van Houten HK, Ziegenfuss JY, Shah ND, Wermers RA, Smith SA. Increased mortality of patients with diabetes reporting severe hypoglycemia. Diabetes Care. 2012;35:1897-901. doi: $10.2337 / d c 11-2054$

[26] Cha SA, Yun JS, Lim TS, Hwang S, Yim EJ, Song KH, et al. Severe Hypoglycemia and Cardiovascular or All-Cause Mortality in Patients with Type 2 Diabetes. Diabetes Metab J. 2016;40:202-10. doi: 10.4093/dmj.2016.40.3.202

[27] Lee AK, Warren B, Lee CJ, McEvoy JW, Matsushita K, Huang ES, et al. The Association of Severe Hypoglycemia With Incident Cardiovascular Events and Mortality in Adults With Type 2 Diabetes. Diabetes Care. 2018;41:104-11. doi: 10.2337/dc17-1669

[28] Kyriacou DN, Lewis RJ. Confounding by Indication in Clinical Research. JAMA. 2016;316:1818-9. doi: 10.1001/jama.2016.16435

[29] Herrett E, Shah AD, Boggon R, Denaxas S, Smeeth L, van Staa T, et al. Completeness and diagnostic validity of recording acute myocardial infarction events in primary care, hospital care, disease registry, and national mortality records: cohort study. BMJ. 2013;346:f2350. doi: $10.1136 / \mathrm{bmj} . \mathrm{f} 2350$

[30] Zaccardi F, Davies MJ, Dhalwani NN, Webb DR, Housley G, Shaw D, et al. Trends in hospital admissions for hypoglycaemia in England: a retrospective, observational study. Lancet Diabetes Endocrinol. 2016;4:677-85. doi: 10.1016/S2213-8587(16)30091-2 
[31] Zaccardi F, Dhalwani NN, Webb DR, Davies MJ, Khunti K. Global burden of hypoglycaemiarelated mortality in 109 countries, from 2000 to 2014: an analysis of death certificates. Diabetologia. 2018;61:1592-602. doi: 10.1007/s00125-018-4626-y 


\section{FIGURE LEGENDS}

Figure 1: Associations of beta-blocker treatment with all-cause death, by presence of hypoglycaemia

Legend: All-cause death hazard ratios, progressively adjusted for potential confounders, are reported comparing any beta-blocker use vs no use in complete-case analysis for patients with (full squares) and without (empty squares) incident hypoglycaemia.

HbA1c: Glycated haemoglobin; LDL-c: Low-density lipoprotein cholesterol; HDL: high-density lipoprotein cholesterol; CVD: cardiovascular disease; SES: socioeconomic status; BMI: body mass index.

Figure 2: Associations of beta-blocker treatment with all-cause death, by presence of hypoglycaemia, after imputation of missing data

Legend: All-cause death hazard ratios, comparing beta-blocker use vs no use, are estimated in the fully-adjusted model (age, sex, smoking, systolic blood pressure, glycated haemoglobin, low-density and high-density lipoprotein cholesterol, prevalent cardiovascular disease, socioeconomic status, body mass index, and Charlson score) for patients with (full squares) and without (empty squares) incident hypoglycaemia. 